\title{
Research on the Broadband Dual-excited Underwater Acoustic Transducer
}

\author{
Kai Zhang ${ }^{1, a}$, Deshi Wang ${ }^{1, a}$, Peng Wang ${ }^{1, b}$, Yiqun $D^{2, c}$ \\ ${ }^{1}$ Department of Weaponry Engineering, Naval University of Engineering, Wuhan430033, China \\ ${ }^{2}$ Institute of Naval Equipment Technology, Beijing, China \\ a406490270@qq.com, bys88488@163.com, 'yichen_wp@163.com
}

Keywords: dual-excited, modal coupling, bandwidth, underwater acoustic transducer

\begin{abstract}
The performance of bandwidth and transmitting voltage response to the piezoelectric dual-excited underwater acoustic transducer are studied by FEM software ANSYS in the paper. At first, the mechanism of the dual-excited transducer is analyzed, once this is done, The FEM models with different axial dimension of the piezoelectric stack and the mid mass, Al and steel are specified for the material of mid mass respectively. the calculated curves are contrasted, the results were found that the appropriate proportion of the mid-mass to the stack will broaden the bandwidth and enhance the transmitting voltage response, the increase of the thickness of the mid-mass will reduce the transmitting voltage response, and the bandwidth can be expanded to $13 \mathrm{kHz}$, the resonance frequency has shifted to a higher value, it ranges from $34 \mathrm{kHz}$ to $46 \mathrm{kHz}$. The results show that one octave bandwidth could be achieved and provide support for the next production and experiment of the broadband underwater acoustic transducer.
\end{abstract}

\section{Introduction}

With the development of acoustic signal processing technology, in order to obtain more information from the target, the transducers with broad band are needed. The conductance curve and the TVR(transmitting voltage response) curve of broadband transducer are flat under the operate frequency. Because of broadening band of the transducers with Single vibrational modal are limited by the mechnical quality factor $Q_{m}$, the doubly resonant or muti-resonant transducer are developing rapidly. Tonpilz acoustic transducer are adopted abroadly by many sonar systems because of its advantage of simple-structure, high-efficient and high electromechanical conversion factor, the studies of broadening its band are researched extensively. There have been many innovative design modification to the basic tonpilz configuration[1,2]. For instance, the use of mechanical head flexure can be employed to broaden the element's bandwidth. Typically, the head mass is designed to be light and stiff with its bending modes placed well above the operating frequency band. yet,exciting the head flexural resonance can produce a second usable frequency band of operation. Another approach to increasing tonpilz bandwidth is the multi-resonant design[3,4]. Here the active drive could consist of two different PZT stacks tuned to two seperated resonance frequencies as showen in fig. 1[5,6]. The stack may be a smart combination of different drive materials as demonstrated by Butler and Tito.

In this paper, the TVR curve and conductance curve of different ratio of two PZT stacks will studied by FEM method. By adjusting the axial dimension of two stacks and mid-mass and checking the material of the mid mass, the bandwidth and TVR performance will be studied.

\section{Mechanism analysis}

Compared with Tonpilz transducer, the structure of dual-excited transducer can be viewed as the vibration system "head mass-spring(1)-mid mass-spring(2)-tail mass", the structure will generate two close resonant points. Dual-excited vibration system with force and velocity transmission is shown in fig. 2. The spring(1) is always a material of passive metal, yielding material or active piezoelectric material. Stiffness $k$ of the spring(2) is less than the spring(1), it controls the lower frequency of the performance. The two parts couple with each other and generate two close harmonic frequencies, and then a needed bandwidth will be achieved in theory[7]. 


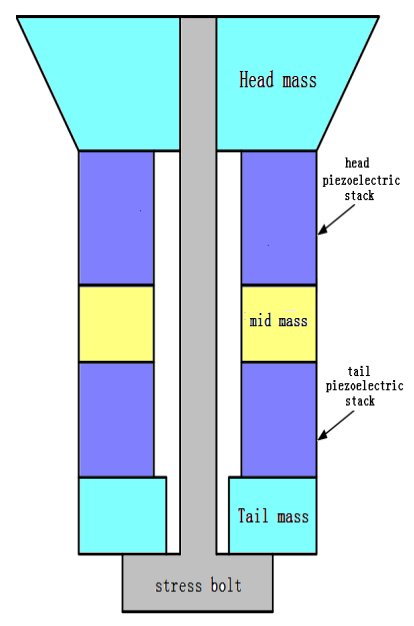

Fig. 1. Structure of dual-excited transducer

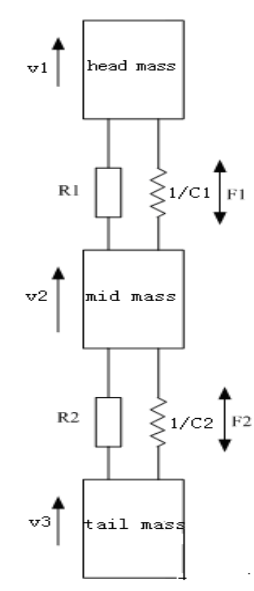

Fig. 2. Dual-excited vibration system

\section{FEM model}

The base of finite element model is entity model. After simplification and approximation, the entity model can represent the transducer closely and can be calculated efficiently. PZT-5A was used as PZT material. Acoustic media is water. The materials of Al and steel are chosed to the mid mass. The axial size of the transducer is fixed, and three different dimensions of mid mass thickness are chosed to $2 \mathrm{~mm}, 4 \mathrm{~mm}$ and $6 \mathrm{~mm}$, thus three ratios of the stacks to mid mass are expressed as 1:10, 1:4.5, 1:2.7, the TVR curve and conductance curve of different dimensions are studied.

Because the dual-excited transducer is axisymmetric, 2-D axisymmetric finite element model is established. PLANE13, PLANE42 and FLUID29 were chose as finite elements for PZT, brass and water. Several layers of water elements adjacent to structure were set incompressible and other water elements were set compressible. FLUID129 was used to simulate the water boundary. Fluid-Structure Interfaces (FSI) was applied on the interface of fluid and structure[8]. Two couple sets of volt were applied on nodes of two surfaces of PZT. The radius of water area shall satisfy far-field condition:

$$
\left\{\begin{array}{l}
r \geq \pi a^{2} / \lambda=\pi a^{2} f / c \\
r \geq a
\end{array}\right.
$$

where $a$ is the radius of cymbal, $\lambda$ is wavelength of sound in water, $c$ is sound velocity in water, $f$ is frequency of sound. Finite element model was generated by mapping method. Considering the precision of calculation, element size was less than $\lambda / 20$. Finite element model of cymbal in water is shown in fig. 3. Problem type was set as harmonic analysis. Damping ratio in water was set as $4.2 \%$. $1 \mathrm{v}$ voltage was applied on upper surface of PZT and 0v on lower surface.

Node electric charge $Q$ and node sound pressure $p$ are included in direct result data of ANSYS. Performance parameters were calculated from these direct result data. Time history post processor can accomplish this work by its equation calculator.

In harmonic analysis,

$$
Q=Q_{0} e^{j \omega t}, \quad I=\frac{d Q}{d t}, \quad V=V_{0} e^{j \omega t}
$$

So admittance is

$$
Y=\frac{I}{V}=j \omega \frac{Q_{0}}{V_{0}}=j 2 \pi f \frac{Q_{0}}{V_{0}} .
$$

When conductance is put as $G$ and susceptance is put as $B$, we get $Y=G+j B$, 
TVR was calculated by

$$
T V R=\operatorname{Re}\left[20 \lg \frac{p r}{V}\right]+120
$$

Whose unit is $\mathrm{dB}$ ref $1 \mu \mathrm{Pa} / \mathrm{V} @ 1 \mathrm{~m}$.

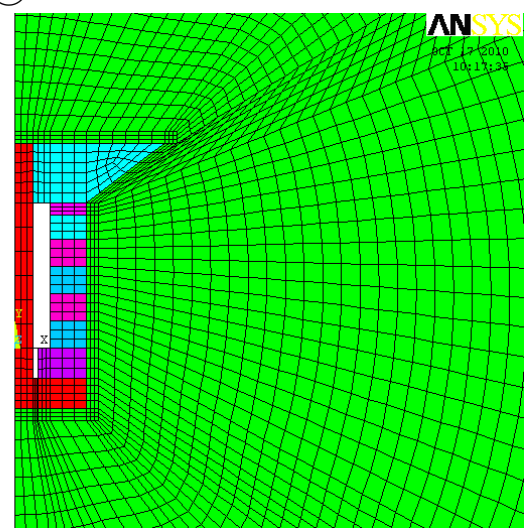

Fig. 3. FEM model of dual-excited transducer

\section{Analysis and argument}

Based on the FEM model, the TVR and conductance are calculated and illustrated in the follow figures. Fig. 4 and fig. 5 show the conductance curve and TVR curve when the material of mid mass is $\mathrm{Al}$, as shown in fig. 4, because of the dual-excited structure, the second harmonic frequency close to the first harmonic frequency, and the notch in the response is flat. With the increase of the ratio value, the peak value of the second resonant frequency increase gradually and the conductance bandwidth increases at the same time. When the value of ratio is 1:2.7, the value of bandwidth is about $13 \mathrm{kHz}$, it increases by about $4 \mathrm{kHz}$. The peak value of TVR has greatly increased with the increase of ratio value.

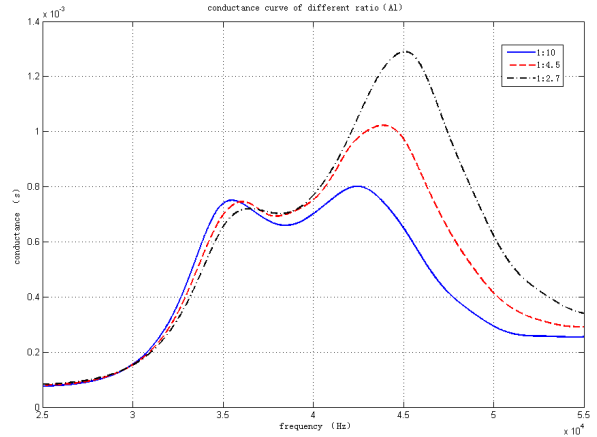

Fig. 4. Conductance curve (Al)

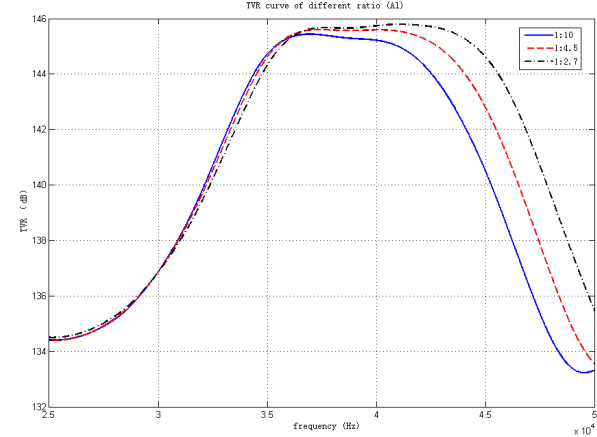

Fig. 5. TVR curve (Al)

Analogously, for the material steel of mid mass, compared with the value of ratio 1:10, the peak value of TVR has increased by $2 \mathrm{~dB}$ when in 1:2.7, and $-3 \mathrm{~dB}$ bandwidth are both kept in about $11 \mathrm{kHz}$. as shown in fig. 6 and fig. 7.

As shown in the Fig. 8 and Fig. 9, The admittance characteristics and transmitting voltage response performance of Dual-excited transducers have changed when the bandwidth are broadened, and the resonance frequency has shifted about $5 \mathrm{kHz}$ to a higher value.

\section{Conclusion}

The bandwidth performance of TVR and conductance to dual-excited transducer are studied, The results are compared and shown that the frequency bandwidth and $-3 \mathrm{~dB}$ bandwidth of transducer can be widen by resonance coupling, the structure can attain an increase of almost one octave bandwidth, the bandwidth of the transducer is about $34 \mathrm{kHz}-46 \mathrm{kHz}$, The admittance characteristics and transmitting voltage response performance of Dual-excited transducers have changed when the 


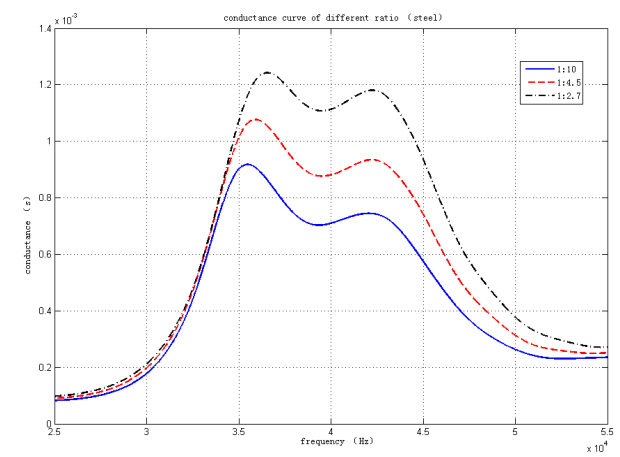

Fig. 6. Conductance curve (steel)

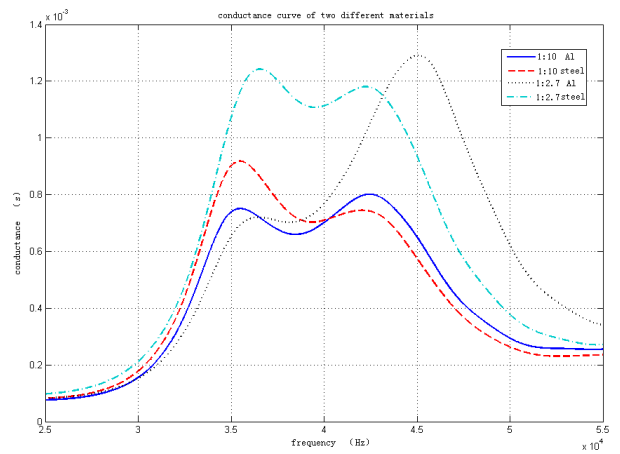

Fig. 8. Conductance curve of different materials

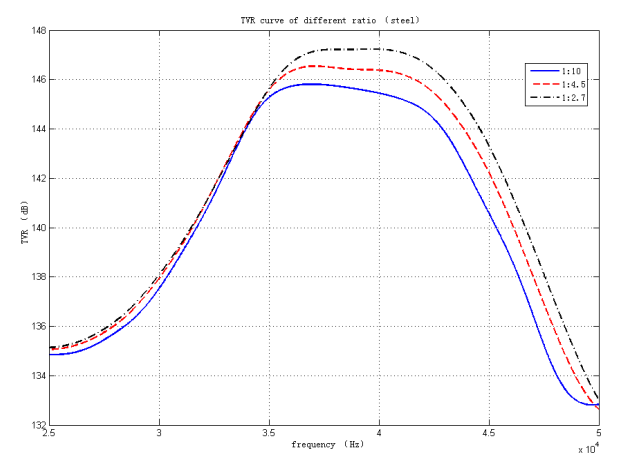

Fig. 7. TVR curve(steel)

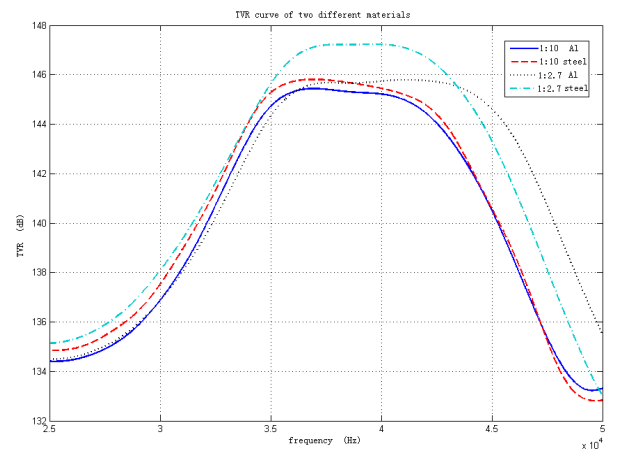

Fig. 9. TVR curve of different materials

bandwidth are broadened,and the resonance frequency has shifted to a higher value, the impacts on the technique index by the material and dimension of the mid-mass are also analyzed and summarized, for the material aluminium of mid-mass, the bandwidth is widened by about $5 \mathrm{kHz}$, and the peak value of transmitting voltage response are reduced by about $2 \mathrm{~dB}$, and for the material steel, the bandwidth is broadened by about $4 \mathrm{kHz}$, the peak value of transmitting voltage response changes slightly. Because of the specific gravity to steel is bigger than aluminium, the above parameters are more sensitive to steel. For another material of the mid-mass can also use the FEM method, and the result will provide technical support to the next production and experiment of the broadband underwater acoustic transducer.

\section{Acknowledgements}

This work was financially supported by Natural Science Foundation of China (Grant No. 51005241).

\section{References}

[1] S.C.Thompson, U.S.Patent 4633119. (1986).

[2] D.Rajapan, Performance of a low-frequency muti-resonant broadband Tonpilz transducer. J. Acoust. Soc. Am. (2002)1692.

[3] S.C.Thompson, M.P.Johnson, Performance and recent developments with doubly resonant wideband transducer. Proc $3^{\text {rd }}$ Int WorkshopTransducers for Sonics and ultransonics. (1992)239-249.

[4] S.C.Butler, Development of a high power broadband doubly resonant transducer (DRT). UDT 2001 conference proceedings, (2001)5-30.

[5] J.L.Butler, S.C.Bulter, Undirectional magnetostrictive/piezoelectric Hybrid transducer. J.Acoust.Soc.Am. (1990)88.

[6] S.C.Butler, F.A.Tito,A broadband hybrid magnetstrictive transducer array, proceedings of the MTS/IEEE Oceans 2000 Conference, 2000

[7] X.P. He, Magnetostrictive transducer, Science press, Beijing, 2006.

[8] X.P.Mo, Simulation and analysis of acoustic transducer using the ANSYS software, Technical Acoustics, 26(2007)1279-1290. 